\title{
Simplified periodic Anderson model: Exact solution in infinite dimensions
}

\author{
R. Consiglio and M. A. Gusmão \\ Instituto de Física, Universidade Federal do Rio Grande do Sul, 91501-970 Porto Alegre, Rio Grande do Sul, Brazil
}

(Received 5 September 1996; revised manuscript received 6 November 1996)

\begin{abstract}
We present a diagrammatic perturbative treatment of the hybridization for the periodic Anderson model that recovers the dynamical mean-field equations in the limit of infinite dimensions. The resulting effective singlesite problem is naturally addressed by perturbation theory on the dynamical mean field. We introduce a simplified version of the model in which only electrons with a given spin orientation hybridize. The perturbation series can be summed in this case, yielding an exact solution for the single-particle Green's functions. Electronic and transport properties are analyzed, showing the existence of a metallic regime with non-Fermiliquid behavior. [S0163-1829(97)03711-9]
\end{abstract}

\section{INTRODUCTION}

Despite the intense research activity of the past decade or two, the physics of heavy-fermion materials still poses interesting challenges. The success of the exactly solved oneimpurity Kondo problem in explaining features that are observed in dilute rare-earth alloys has not been matched in the study of concentrated Kondo systems. ${ }^{1,2}$ In addition to a high-temperature behavior more or less consistent with independent magnetic impurities, these systems present a lowtemperature coherent regime, where the resistivity drops down to a very small residual value. In this region, the system can behave as a normal Fermi liquid with enhanced effective mass, or undergo a phase transition to a superconducting or a magnetically ordered state, with possible coexistence of the two.

It is generally accepted that the relevant physical ingredients of the problem are contained in the so-called periodic Anderson model (PAM), or Anderson lattice. This model describes a noninteracting conduction band that hybridizes with localized levels (intended to represent $f$ levels of rareearth ions). A strong on-site Coulomb repulsion reduces the probability of double occupancy of these levels. For simplicity, the hybridization is usually considered as a local process. In addition, orbital degeneracy is often neglected, which would be justified by a strong crystal-field splitting of the levels. In contrast to the single-impurity model, ${ }^{3}$ there is no exact solution of the Anderson lattice, and many approximate treatments have been developed in the past. ${ }^{4,5}$ Different approaches have focused on distinct aspects of the problem, but none of them has been able to produce a complete coherent picture.

The limit of infinite spatial dimensionality for fermions on a lattice, introduced by Metzner and Vollhardt, ${ }^{6}$ has attracted much interest due to the simplifications that it brings to the analysis of strongly correlated systems. Similarly to what has been done for the Hubbard model, self-consistent equations for the Green's functions of an effective single-site problem in the presence of a dynamical mean field have been obtained for the Anderson lattice. ${ }^{7,8}$ Even though the selfconsistency equations are exact in $d=\infty$, one still has to solve the effective single-site problem. Numerical solutions for both the Hubbard and Anderson models have been ob- tained, and analytical approximate solutions based on expansion on the Coulomb interaction have also been worked out (see Ref. 9 and references therein).

Starting with perturbation theory on the hybridization term, we recover here the exact dynamical mean-field equations for the periodic Anderson model in infinite spatial dimensionality. In addition, the perturbative series on the hybridization term is naturally transformed in this limit into a perturbative series on the local dynamic mean field. Due to the inclusion of the Coulomb interaction in the unperturbed Hamiltonian, this perturbation series constitutes a strongcoupling approach to the problem that may complement weak-coupling methods as far as analytical solutions are concerned. Of course, it is not possible to sum the entire perturbation series, and all analytic solutions are approximate. However, in a similar treatment ${ }^{10,11}$ the corresponding series can be summed for the Falicov-Kimball (FK) model, ${ }^{12}$ which can be viewed as a simplified Hubbard model, and has an exact solution. ${ }^{13,14}$ In analogy with this, we introduce here a simplified periodic Anderson model (SPAM) in which only $f$ and $c$ electrons with a given spin orientation hybridize. For this model we were able to calculate exactly the relevant Green's functions, using them to study spectral and transport properties. We restrict our analysis to the paramagnetic state, and remain in the half-filling case, related to the physics of Kondo insulators. ${ }^{15}$

The exact solution of the SPAM in $d=\infty$ shows some expected features of the physics of heavy-fermion materials, although some important ones are missing. For instance, the single-particle density of states (DOS) shows narrow $f$ bands resulting from the broadening of the initially localized $f$ levels, and a hybridization gap opens in the middle of the conduction band for sufficiently large hybridization, but the expected Kondo resonance at the Fermi level is absent. When there is no gap, the low-frequency optical conductivity is enhanced at low temperatures, although a true Drude peak is not formed. Consistent with this, the static resistivity drops down in a low-temperature "quasicoherent" regime. The resistance varies linearly with temperature in this region, and has a finite zero-temperature limit, showing that the system is not in a Fermi-liquid state.

In Sec. II we write down the Hamiltonian for the PAM, and introduce the relevant Green's functions and their calcu- 
lation by means of a perturbation expansion on the hybridization term. The infinite dimension limit is addressed in Sec. III. In Sec. IV we introduce the simplified periodic Anderson model, and discuss its exact solution. Our final remarks and conclusions appear in Sec. V.

\section{MODEL AND PERTURBATION APPROACH}

The basic version of the periodic Anderson model, without orbital degeneracy and with a local hybridization, can be represented by the model Hamiltonian

$$
\begin{aligned}
H= & -t \sum_{\langle i j\rangle \sigma} c_{i \sigma}^{\dagger} c_{j \sigma}+E \sum_{i \sigma} n_{i \sigma}^{f}+U \sum_{i} n_{i \uparrow}^{f} n_{i \downarrow}^{f} \\
& +V \sum_{i \sigma}\left(c_{i \sigma}^{\dagger} f_{i \sigma}+f_{i \sigma}^{\dagger} c_{i \sigma}\right),
\end{aligned}
$$

in usual notation. We have chosen to write the conduction band in a tight-binding form, with a nearest-neighbor hopping amplitude $t$, because this will allow extension to the infinite dimension limit. The first three terms on the righthand side of Eq. (1) will be considered as the unperturbed Hamiltonian $H_{0}$, which is a sum of a local and a band terms that can be solved independently. Thus, the unperturbed basis states span the product of two decoupled spaces: the local eigenstates of the $f$-electron Hamiltonian, and the conduction band Bloch states. The last term in Eq. (1) will be taken as the perturbation.

We will be interested in the temperature-dependent oneparticle Green's functions

$$
G_{i j \sigma}^{c}(\tau) \equiv-\left\langle\hat{T} c_{i \sigma}(\tau) c_{j \sigma}^{\dagger}(0)\right\rangle
$$

and

$$
G_{i j \sigma}^{f}(\tau) \equiv-\left\langle\hat{T} f_{i \sigma}(\tau) f_{j \sigma}^{\dagger}(0)\right\rangle .
$$

We use a diagrammatic representation of the perturbation series for these functions in which there are vertices corresponding to local cumulant averages of $f$ operators, and lines representing the unperturbed Green's function of the conduction band, with a factor $V$ associated with each "contact" between a line and a vertex. ${ }^{16}$ It is possible to define an irreducible $f$-electron Green's function, that we will represent (in wave vector and Matsubara's frequency space) by $\mathcal{G}_{\mathbf{k} \sigma}\left(i \omega_{n}\right)$, which is the sum of all diagrams that are not separated in two disconnected parts after a single line is cut. Some example diagrams of this function are shown in Fig. 1. It is straightforward to show that the perturbation series for both conduction and $f$-electron Green's functions can be formally summed in the form of Dyson-like equations:

$$
G_{\mathbf{k} \sigma}^{c}\left(i \omega_{n}\right)=g_{\mathbf{k} \sigma}^{c}\left(i \omega_{n}\right)+g_{\mathbf{k} \sigma}^{c}\left(i \omega_{n}\right) V \mathcal{G}_{\mathbf{k} \sigma}\left(i \omega_{n}\right) V G_{\mathbf{k} \sigma}^{c}\left(i \omega_{n}\right)
$$

and

$$
G_{\mathbf{k} \sigma}^{f}\left(i \omega_{n}\right)=\mathcal{G}_{\mathbf{k} \sigma}\left(i \omega_{n}\right)+\mathcal{G}_{\mathbf{k} \sigma}\left(i \omega_{n}\right) V g_{\mathbf{k} \sigma}^{c}\left(i \omega_{n}\right) V G_{\mathbf{k} \sigma}^{f}\left(i \omega_{n}\right),
$$

where $g_{\mathbf{k} \sigma}^{c}\left(i \omega_{n}\right)=1 /\left(i \omega_{n}+\mu-\varepsilon_{\mathbf{k}}\right)$ is the unperturbed conduction-electron Green's function, $\varepsilon_{\mathbf{k}}$ being the tightbinding energies of the unhybridized conduction band, which

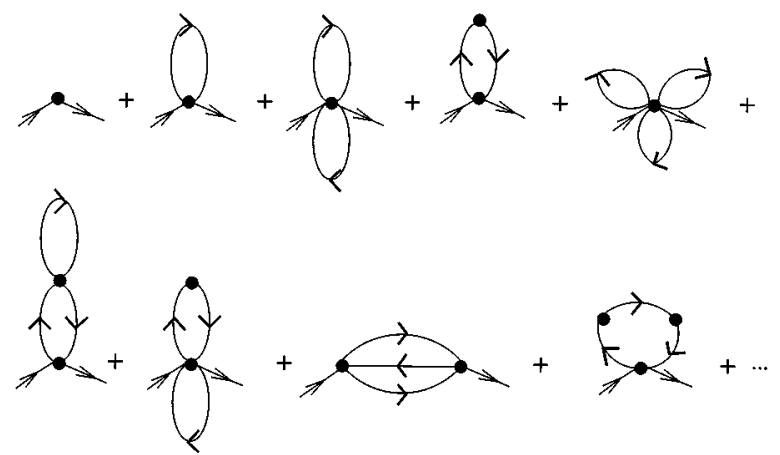

FIG. 1. Some representative diagrams of the $f$-electron irreducible Green's function. The vertices (dots) are cumulant averages of $f$-electron creation and annihilation operators, and the internal lines represent conduction-electron unperturbed Green's functions (the external lines are just a guide for the eye).

assume the form $\varepsilon_{\mathbf{k}}=-2 t \Sigma_{\mu=1}^{d} \cos k_{\mu}$, for a hypercubic lattice in $d$ dimensions (with a unit lattice parameter). Equation (5) has precisely the same structure found for the Green's function in the Hubbard model when the hopping term is treated as a perturbation, ${ }^{10}$ provided we substitute $V^{2} g_{\mathbf{k} \sigma}^{c}\left(i \omega_{n}\right)$ for the tight-binding energies of that model. With this, all formal relations obtained for the Hubbard case can be employed here to the $f$ electrons, with the replacement just mentioned. We will make full use of this in the next sections. Equations (4) and (5) allow us to write

$$
G_{\mathbf{k} \sigma}^{c}\left(i \omega_{n}\right)=\frac{1}{i \omega_{n}-\varepsilon_{\mathbf{k}}+\mu-V^{2} \mathcal{G}_{\mathbf{k} \sigma}\left(i \omega_{n}\right)}
$$

and

$$
G_{\mathbf{k} \sigma}^{f}\left(i \omega_{n}\right)=\mathcal{G}_{\mathbf{k} \sigma}\left(i \omega_{n}\right)+\mathcal{G}_{\mathbf{k} \sigma}\left(i \omega_{n}\right) V G_{\mathbf{k} \sigma}^{c}\left(i \omega_{n}\right) V \mathcal{G}_{\mathbf{k} \sigma}\left(i \omega_{n}\right) .
$$

We have written the last equations in a convenient form to apply the infinite-dimension formalism to be developed next.

\section{THE INFINITE DIMENSION LIMIT}

When considering the limit $d \rightarrow \infty$, in order to preserve a finite energy per particle in the conduction band, one has to scale the hopping amplitude ${ }^{6}$ such that $d t^{2}=$ const. Here we choose $4 d t^{2} \equiv t^{* 2}=1$, which means that all energies will be measured in units of $t^{*}$.

In this limit, the diagrammatic series is greatly simplified. Equivalently to what has been shown for the Hubbard model, ${ }^{11}$ only the site-diagonal part of the irreducible Green's function $\mathcal{G}_{\sigma}\left(i \omega_{n}\right) \equiv(1 / N) \sum_{\mathbf{k}} \mathcal{G}_{\mathbf{k} \sigma}\left(i \omega_{n}\right)$ survives in the limit $d=\infty$, and only diagrams with independent loops, as shown in Fig. 2, are nonvanishing. The site-diagonal Green's function for the $f$ electrons, $G_{i i \sigma}^{f}\left(i \omega_{n}\right)$ can also be evaluated from the same set of diagrams as $\mathcal{G}_{\sigma}\left(i \omega_{n}\right)$, with a slight reinterpretation of each diagram. ${ }^{11}$ It is then clear from the diagrammatic series that the problem of obtaining the site-diagonal $f$-electron Green's function has been reduced to a single-site problem. The loops that go in and out of the vertex corresponding to this site can be viewed as a local dynamic mean-field connecting the site to a particle bath (the rest of the lattice). Since an electron leaves or enters a 


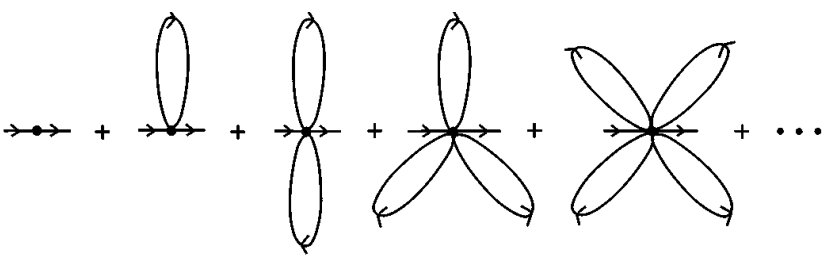

FIG. 2. Diagrammatic series for the irreducible Green's function $\mathcal{G}_{\sigma}$ or the site-diagonal Green's function $G_{i i \sigma}^{f}$ in $d=\infty$. Each line forming a closed loop represents a fully renormalized conductionelectron Green's function in the case of $\mathcal{G}_{\sigma}$, or the dynamic mean field $\mathcal{A}_{\sigma}\left(i \omega_{n}\right)$ for $G_{i i \sigma}^{f}$.

local $f$ level through a hybridization process that takes it to or brings it from the conduction band, at first sight the local field should be $\widetilde{\mathcal{A}}_{\sigma}\left(i \omega_{n}\right) \equiv V^{2} G_{i i \sigma}^{c}$. However, when going from a local cumulant average to a regular one we subtract all "visits" to the original site in the renormalization of the conduction-electron Green's function. The true local field, that we represent here by $\mathcal{A}_{\sigma}\left(i \omega_{n}\right)$, can then be written as

$\widetilde{\mathcal{A}}_{\sigma}=\mathcal{A}_{\sigma}+\mathcal{A}_{\sigma} \mathcal{G}_{\sigma} \mathcal{A}_{\sigma}+\mathcal{A}_{\sigma} \mathcal{G}_{\sigma} \mathcal{A}_{\sigma} \mathcal{G}_{\sigma} \mathcal{A}_{\sigma}+\cdots=\left\{\mathcal{A}_{\sigma}^{-1}-\mathcal{G}_{\sigma}\right\}^{-1}$.

Self-consistency is achieved through the Fourier transform, by imposing that quantities calculated directly for the effective single-site problem are identical to the corresponding ones for the lattice. For instance, Eqs. (6) and (7) yield

$$
G_{i i \sigma}^{c}\left(i \omega_{n}\right)=\frac{1}{N} \sum_{\mathbf{k}} \frac{1}{i \omega_{n}+\mu-V^{2} \mathcal{G}_{\sigma}\left(i \omega_{n}\right)-\varepsilon_{\mathbf{k}}}
$$

and

$$
G_{i i \sigma}^{f}\left(i \omega_{n}\right)=\mathcal{G}_{\sigma}\left(i \omega_{n}\right)\left[1+\mathcal{G}_{\sigma}\left(i \omega_{n}\right) V^{2} G_{i i \sigma}^{c}\left(i \omega_{n}\right)\right] .
$$

From Eqs. (8) and (10) and the definition of $\widetilde{\mathcal{A}}_{\sigma}$ one obtains

$$
\mathcal{A}_{\sigma}=\mathcal{G}_{\sigma}^{-1}-\left[G_{i i \sigma}^{f}\right]^{-1} .
$$

Equations (9)-(11), together with an explicit solution of the single-site problem for $G_{i i \sigma}^{f}\left(i \omega_{n}\right)$ in terms of $\mathcal{A}_{\sigma}\left(i \omega_{n}\right)$, constitute a self-consistent set of equations for all the relevant Green's functions and the local dynamic mean field. Notice that from Eqs. (5) and (11) we can write

$$
G_{i i \sigma}^{f}=\frac{1}{N} \sum_{\mathbf{k}} \frac{1}{\mathcal{G}_{\sigma}^{-1}-V^{2} g_{\mathbf{k} \sigma}^{c}}=\frac{1}{\mathcal{G}_{\sigma}^{-1}-\mathcal{A}_{\sigma}},
$$

where the role of $\mathcal{A}_{\sigma}$ as a mean field is made explicit.

Since all $\mathbf{k}$ dependence is now restricted to the tightbinding energies $\varepsilon_{\mathbf{k}}$, we can replace the sum over $\mathbf{k}$ in Eq. (9) by an energy integral in the form

$$
G_{i i \sigma}^{c}\left(i \omega_{n}\right)=\int d \varepsilon \frac{D_{0}(\varepsilon)}{i \omega_{n}+\mu-V^{2} \mathcal{G}_{\sigma}\left(i \omega_{n}\right)-\varepsilon},
$$

where $D_{0}(\varepsilon)$ is the uncorrelated density of states of the conduction band in $d=\infty$. For a hypercubic lattice (with the choice of $t^{*}=1$ that we have made) one has ${ }^{6}$ $D_{0}(\varepsilon)=(1 / \sqrt{\pi}) \exp \left(-\varepsilon^{2}\right)$.

Our method yields an approach to solve the single-site effective problem as a perturbation series in the fields $\mathcal{A}_{\sigma}$ (see Fig. 2). Starting with the unperturbed local Green's function $-\left\langle\hat{T} f_{\sigma}(\tau) f_{\sigma}^{\dagger}(0)\right\rangle_{0}$, each even order in perturbation theory introduces a product of the type $\mathcal{A}_{\sigma_{1}}\left(\tau_{1}-\tau_{1}^{\prime}\right) f_{\sigma_{1}}^{\dagger}\left(\tau_{1}\right) f_{\sigma_{1}}\left(\tau_{1}^{\prime}\right)$, so that in general one has to calculate averages of the form $\left\langle f_{\sigma_{1}}^{\dagger}\left(\tau_{1}\right) f_{\sigma_{1}}\left(\tau_{1}^{\prime}\right) f_{\sigma_{2}}^{\dagger}\left(\tau_{2}\right) f_{\sigma_{2}}\left(\tau_{2}^{\prime}\right) \cdots\right\rangle_{0}$. Since this is exactly the same as in the Hubbard model, we are not going to discuss it here, and we refer the reader to Ref. 11 .

\section{Dynamic and static conductivity}

Now we briefly review the calculation of the frequencydependent conductivity for the Anderson model in infinite dimensions. The conductivity tensor in linear response theory is given by Kubo's formula in terms of the currentcurrent correlation function. Due to the absence of hopping for the $f$ electrons in the Anderson model, the current operator refers to the conduction electrons only. In infinite dimensions the difficulties related to momentum conservation disappear, and the optical conductivity (reduced to a scalar due to the hypercubic symmetry) assumes the simple form ${ }^{17}$

$$
\begin{aligned}
\sigma(\nu)= & \pi \sum_{\sigma} \int d \varepsilon D_{0}(\varepsilon) \int d \omega A_{\sigma}(\varepsilon, \omega) A_{\sigma}(\varepsilon, \omega+\nu) \\
& \times \frac{[f(\omega)-f(\omega+\nu)]}{\nu},
\end{aligned}
$$

where $f(\epsilon)$ is the Fermi function and $A_{\sigma}(\varepsilon, \omega)$ is the oneparticle spectral density, obtained by replacing $\varepsilon_{\mathbf{k}}$ by $\varepsilon$ in

$$
A_{\sigma}\left(\varepsilon_{\mathbf{k}}, \omega\right) \equiv-\frac{1}{\pi} \operatorname{Im} G_{\mathbf{k} \sigma}^{c}\left(\omega+i 0^{+}\right)
$$

where we perform the analytic continuation of the conduction-electron Green's function, Eq. (6), to real frequencies.

One can obtain the static limit $(\nu \rightarrow 0)$ of Eq. (14),

$$
\sigma(0)=\pi \beta \int d \varepsilon D_{0}(\varepsilon) \int d \omega[A(\varepsilon, \omega)]^{2} f(\omega)[1-f(\omega)]
$$

where $\beta \equiv 1 / T$ stands for the inverse of the temperature. From Eq. (16) the static resistivity $\rho \equiv 1 / \sigma(0)$ can also be calculated.

\section{THE SIMPLIFIED PERIODIC ANDERSON MODEL}

Inspired by the fact that the perturbation series in $\mathcal{A}_{\sigma}$ for the effective single-site problem in $d=\infty$ can be exactly summed in the case of the Falicov-Kimball model, we introduce here a similar simplification of the Anderson model that will allow us to find an exact solution. The periodic Anderson model can be simplified by considering a spin-dependent hybridization such that only electrons with a given spin orientation (say, spin up) hybridize. In this case, the Hamiltonian (1) can be rewritten in the form 

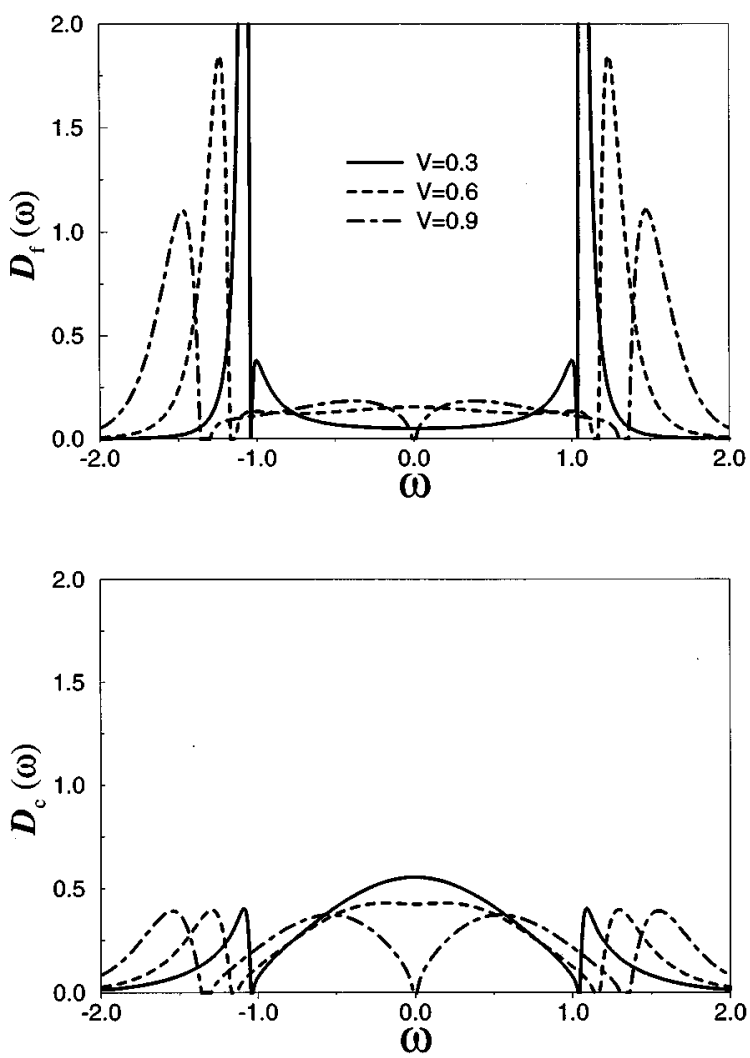

FIG. 3. Densities of states for the $f$ and $c$ electrons (top and bottom, respectively) in the half-filled SPAM in infinite dimension for $U=2$ and some values of $V$.

$$
\begin{aligned}
H= & -t \sum_{\langle i j\rangle \sigma} c_{i \sigma}^{\dagger} c_{j \sigma}+E \sum_{i \sigma} n_{i \sigma}^{f}+U \sum_{i} n_{i \uparrow}^{f} n_{i \downarrow}^{f} \\
& +V \sum_{i}\left(c_{i \uparrow}^{\dagger} f_{i \uparrow}+f_{i \uparrow}^{\dagger} c_{i \uparrow}\right) .
\end{aligned}
$$

Actually, the dynamics of the spin-down conduction electrons is completely decoupled from that of the other electrons, and they behave as a free gas. However, they are thermodynamically coupled to the system, and their number per lattice site is not a priori fixed. Here we will only consider the paramagnetic, half-filled case in the particle-hole symmetric situation $(E=-U / 2)$. This fixes the chemical potential at $\mu=0$, and we will completely neglect the existence of the spin-down conduction electrons.

As far as the $f$ electrons are concerned, we are faced with exactly the same problem as in the Falicov-Kimball model. Summation of the perturbation series in $\mathcal{A}_{\uparrow}$ yields

$$
\begin{aligned}
G_{i i \uparrow}^{f}\left(i \omega_{n}\right)= & \frac{1-\left\langle n_{\downarrow}^{f}\right\rangle}{i \omega_{n}-E+\mu-\mathcal{A}_{\uparrow}\left(i \omega_{n}\right)} \\
& +\frac{\left\langle n_{\downarrow}^{f}\right\rangle}{i \omega_{n}-E-U+\mu-\mathcal{A}_{\uparrow}\left(i \omega_{n}\right)} .
\end{aligned}
$$

For the case that we are considering, this reduces to the simple form
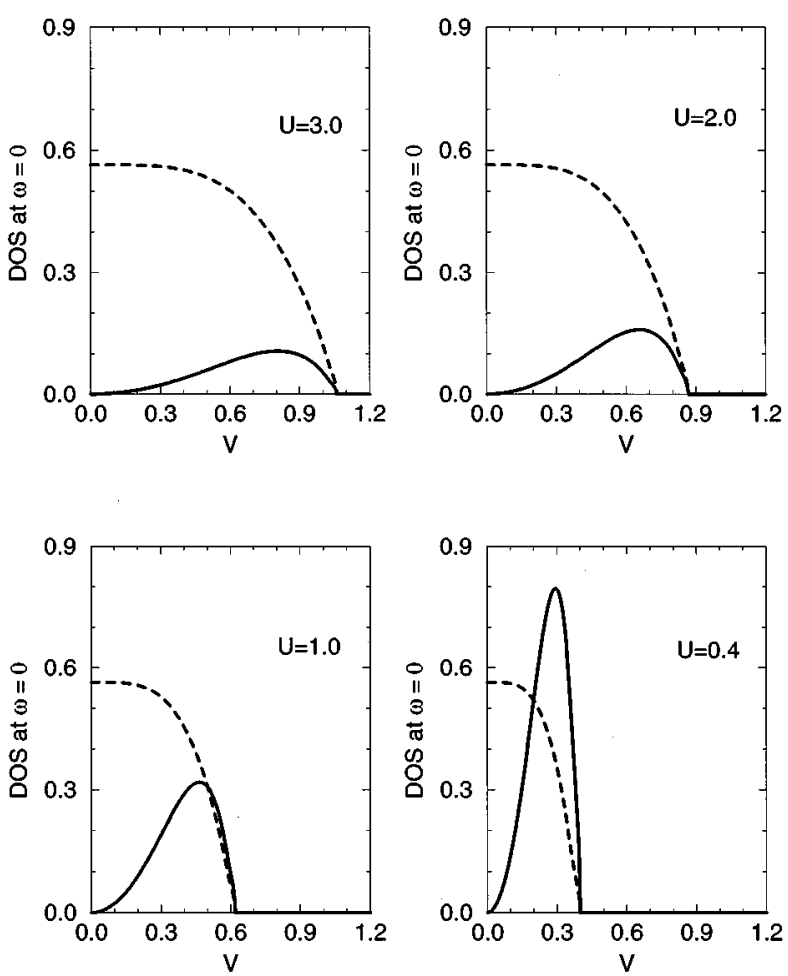

FIG. 4. Densities of states at the Fermi level for $f$ (full) and $c$ (dotted) electrons in the SPAM as a function of the hybridization parameter for some values of $U$.

$$
G_{i i \uparrow}^{f}\left(i \omega_{n}\right)=\frac{1 / 2}{i \omega_{n}+U / 2-\mathcal{A}_{\uparrow}\left(i \omega_{n}\right)}+\frac{1 / 2}{i \omega_{n}-U / 2-\mathcal{A}_{\uparrow}\left(i \omega_{n}\right)} .
$$

The self-consistent solution of Eqs. (10), (11), (13), and (19) is easily performed numerically. We can extend these equations to real frequencies by the direct substitution $i \omega_{n} \rightarrow \omega+i 0^{+}$, which allow us to obtain single-particle densities of states, $D_{\alpha}(\omega)=-(1 / \pi) \operatorname{Im} G_{i i \uparrow}^{\alpha}\left(\omega+i 0^{+}\right)$, where $\alpha$ refers to $c$ or $f$ electrons.

\section{Results}

As far as electronic properties are concerned, our main results are presented in Figs. 3 and 4. As we mentioned before, we have restricted our analysis to the paramagnetic state in the half-filling case. In Fig. 3 we show densities of states for the (spin-up) $f$ and $c$ electrons for $U=2$ and some values of $V$. One can see that the very narrow peaks that mark the singly and doubly occupied localized $f$ levels are broadened and reduced in height as the hybridization is increased. Spectral weight is displaced from these satellite peaks to the central region of the spectrum. On the other hand, the conduction-band spectral weight is partially shifted to the satellite peaks. Eventually, for large enough $V$, a gap opens in the center of the band. As $V$ increases from zero, the $f$-electron DOS at the Fermi level first increases, reaches a maximum, and then decreases towards zero when the gap appears. This is best seen in Fig. 4, where it is clear that the critical value of $V$ for which the gap appears approaches zero as $U$ is lowered. Of course, for $V$ strictly zero the two bands are decoupled, and there is no gap. The most striking con- 

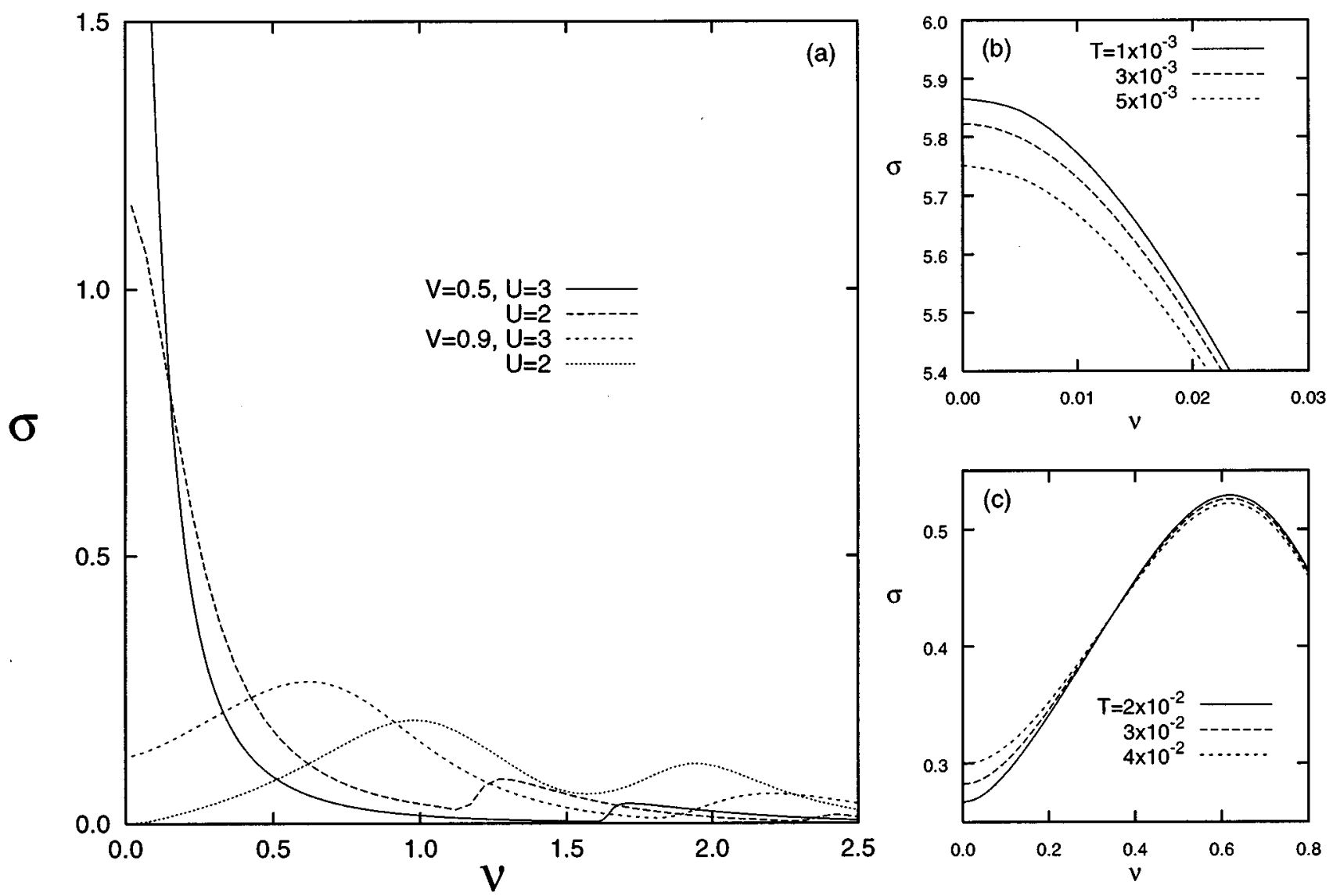

FIG. 5. Frequency dependence of the optical conductivity of the symmetric SPAM. (a) General behavior for $T=1.0 \times 10^{-3}$ and some values of $V$ and $U$. (b) Detailed view of the low-frequency region with varying temperature (for $U=3.0$ and $V=0.5$ ) showing the absence of a Drude peak. (c) Low-frequency region for large hybridization $(V=0.9)$, where $\sigma$ increases with temperature, in contrast with the behavior observed in (b).

trast between the DOS for the SPAM and numerical results for the complete Anderson model ${ }^{9}$ is the absence of a Kondo peak at the Fermi level with a hybridization gap in the middle of it. The enhancement of the DOS at the Fermi level is weak, and the hybridization gap is absent for certain ranges of parameters (large $U$, low $V$ ).

The frequency-dependent conductivity, shown in Fig. 5, is consistent with the electronic structure, reflecting the gap at the Fermi level when it exists, and showing a finite value at zero frequency when the system is a conductor. Notice that the conductivity enhancement observed in the low-frequency region in Fig. 5(a) is not the beginning of a Drude peak: the limiting value at zero temperature and frequency is always finite, as can be seen in Fig. 5(b). This limiting value is lowered by increasing temperature in the low-hybridization regime. In contrast, for large values of $V$ the conductivity is depleted in the low-frequency region, and its limiting value increases with temperature [Fig. 5(c)]. This reflects the existence of a pseudogap in the density of states, as can be seen in Fig. 3. Thermal excitation of carriers across the Fermi surface enhances the conductivity in this situation.

A complementary view of the conduction properties in the zero-frequency limit is provided by the static resistivity (Fig. 6), which shows a low-temperature "quasicoherent" regime, with an approximately linear decrease towards a nonzero limit, signaling a breakdown of Fermi-liquid behavior in the SPAM. This is consistent with the fact that the self-energy of the spin-up conduction electrons remains finite at zero frequency, and does not depend on temperature. The selfenergy is just given by $V^{2} \mathcal{G}_{\uparrow}$, as can be seen by the form of $G_{\mathbf{k} \sigma}^{c}$, Eq. (6). Notice that the temperature dependence of the resistivity in the SPAM comes exclusively from the explicit factor $\beta$ and the Fermi functions in Eq. (16). The Green's functions depend on temperature only through the Matsubara frequencies, and this dependence disappears upon analytic
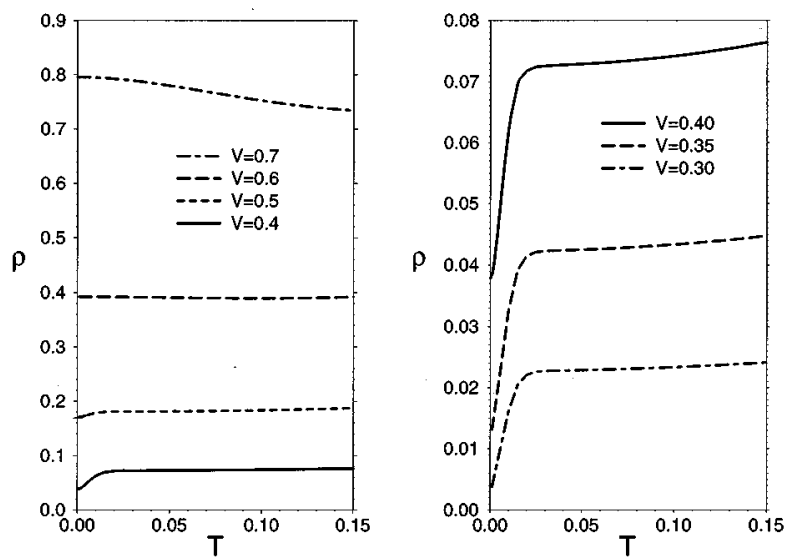

FIG. 6. Temperature dependence of the static resistivity for $U=3$ and various values of $V$. Left: change in behavior with hybridization. Right: detailed view of the low- $V$ regime. 
continuation to real frequencies. Of course, the spectral function in Eq. (16) plays an important role in defining the relevant integration region, with an (indirect) effect on the temperature dependence of the conductivity. The temperature independence of real-frequency Green's functions is a characteristic of the SPAM, since the "loops" in Fig. 2 are not really closed: the only frequency going around all of them is the external one. The situation would change for the complete PAM, when summation over internal Matsubara frequencies in the loops should produce a temperature dependence that could survive the analytic continuation to real frequencies.

It is interesting to compare our results for the optical conductivity or static resistivity with experimental observations in $f$-electron materials that show non-Fermi-liquid ground states. ${ }^{18,19}$ This happens for Kondo alloys, such as $\mathrm{Y}_{1-x} \mathrm{U}_{x} \mathrm{Pd}_{3}$ or $\mathrm{Y}_{1-x-y} \mathrm{Th}_{y} \mathrm{U}_{x} \mathrm{Pd}_{3}$. Depending on the concentration parameters, the resistivity versus temperature plots can show the same kind of changes of the general trend as observed in Fig. 6, where the parameter that is varied is the hybridization strength. As a possible explanation for such a similarity, we must notice that the SPAM (at least for nonmagnetic phases, as considered here) has an intrinsic disorder built into it, since the frozen spin-down $f$ electrons act as randomly distributed scattering centers. We have fixed the concentration of these frozen electrons by fixing the band filling. So, the strength of their effect on the conduction electrons depends on the hybridization: it is only through their acquired $f$ character that the moving electrons can interact with the frozen ones via the Coulomb repulsion. In real compounds the effectiveness of the scattering mechanism is governed by the concentration of impurities. Thus, varying the hybridization in the model or the concentration of impurities in real materials amounts to varying the scattering rate of conduction electrons, which explains the similarity between the resistivity behavior with temperature in both cases. Even though a quantitative comparison would not be justifiable here, we can at least check orders of magnitude. Our units are such that energy, frequency, and temperature are all measured in units of $t^{*}$, which is essentially one-half of the bandwidth. On the other hand, our conductivity must be multiplied by $t^{* 2}$. Choosing a bandwidth of $\sim 1 \mathrm{eV}$, and inserting all the relevant universal constants we obtain temperatures between 0 and $1000 \mathrm{~K}$, a unit frequency corresponds to $\sim 10^{4} \mathrm{~cm}^{-1}$, and our conductivities are of the order of $10^{3}(\Omega \mathrm{cm})^{-1}$. This is more or less the right order of magnitude to compare with the experimental results. Of course, changes by a factor of 1 to 10 can be produced by other choices of parameter values.

\section{CONCLUSIONS}

In conclusion, we have presented a diagrammatic method to handle the perturbation series in the hybridization term for one-particle Green's functions of the Anderson lattice. All the relevant equations can be written in terms of an irreducible $f$-electron Green's function, whose diagrammatic representation can be formally related to the corresponding one for the Hubbard model treated by perturbation around the atomic limit. ${ }^{11}$ With this, the reduction to a single-site effective problem in $d=\infty$ is easily obtained. Besides reproducing the exact self-consistency equations satisfied by the Green's functions of both kinds of electrons and the local dynamic mean-field, ${ }^{9}$ the method provides a perturbation series on this mean field for the site-diagonal $f$-electron Green's function. We have introduced a simplified version of the periodic Anderson model for which this perturbation series is exactly summable. Inspired by the relationship between the FalicovKimball and Hubbard models, we defined the SPAM by eliminating the hybridization of down-spin electrons in the Anderson model. We want to emphasize that the SPAM cannot be reduced to the Falicov-Kimball model, even though we defined the former in analogy with the latter. Also, although the tight-binding solution of the Hubbard model in infinite dimensions has been used here to deal with the diagrammatic representation of the $f$-electron Green's functions, the self-consistency conditions are different, reflecting the existence of the conduction band, which leads to different solutions.

A summary of the exact results that we have obtained for the SPAM is as follows: There is broadening of the localized $f$ levels, with exchange of spectral weight between the lowand high-energy regions of the spectrum. Above a critical value of $V$ (which increases with increasing $U$ ) a hybridization gap opens up at the center of the conduction band. A Kondo peak does not appear. For small values of the hybridization parameter, the optical conductivity is enhanced in the low-frequency region, but a true Drude peak is absent. The finite zero-frequency value of the conductivity is reduced with increasing temperature in this regime. We have thus a metallic regime where the static resistivity shows a sharp drop with temperature, but the system is not a Fermi liquid: the dependence of the resistivity with temperature is approximately linear, and there is a finite residual resistance. When the hybridization parameter is large, as one approaches the limiting value for the formation of a hybridization gap, there is a reversal of the temperature behavior of the lowfrequency conductivity due to the contribution of thermal excitations across a pseudogap appearing in the density of states. The overall features of the conductivity behavior with temperature in the SPAM are qualitatively similar to what is experimentally observed in disordered heavy-fermion systems such as $\mathrm{Y}_{1-x} \mathrm{U}_{x} \mathrm{Pd}_{3}$ and related compounds. ${ }^{18}$ This similarity can be traced back to the existence of an intrinsic disorder in the model due to the presence of randomly distributed scattering centers: the (nonmobile) down-spin $f$ electrons.

Up to now, we have restricted our calculation to the particle-hole symmetric case in the paramagnetic phase. It should be interesting to investigate the existence of ordered phases, as well as the spectral and transport properties for other band fillings. Although the breakdown of spinsymmetry inherent to the simplified Anderson model is highly non-physical, the fact that there is an exact solution of the problem is very important. In particular, this solution can be a checking point for approximation schemes devised for the complete model. For instance, the exactly calculated quantities for up-spin electrons in the SPAM can be considered as a reasonable approximation for electrons with both spin orientations in the PAM. The simplified model could, then, be a starting point for studies that included corrections 
taking into account the hybridization of the other spin species.

\section{ACKNOWLEDGMENTS}

We are indebted to Luis Craco for his valuable help with the computation routines, as well as for interesting comments and discussions. We acknowledge support by the Brazilian agencies Conselho Nacional de Desenvolvimento Científico e Tecnológico (CNPq), Financiadora de Estudos e Projetos (FINEP), and Fundação de Amparo à Pesquisa do Estado do Rio Grande do Sul (FAPERGS). R.C. thanks Fundação Universidade do Rio Grande (Brazil) for providing conditions that allowed his participation in this work to begin.
${ }^{1}$ A. C. Hewson, The Kondo Problem to Heavy Fermions (Cambridge University Press, Cambridge, 1993).

${ }^{2}$ G. R. Stewart, Rev. Mod. Phys. 56, 755 (1984).

${ }^{3}$ P. B. Wiegmann, J. Phys. C 14, 1463 (1981).

${ }^{4}$ G. Czycholl, Phys. Rep. 143, 277 (1986).

${ }^{5}$ P. A. Lee, T. M. Rice, J. W. Serene, L. J. Sham, and J. W. Wilkins, Comments Condens. Matter Phys. 12, 99 (1986).

${ }^{6}$ W. Metzner and D. Vollhardt, Phys. Rev. Lett. 62, 324 (1989); E. Müller-Hartmann, Z. Phys. B 74, 507 (1989). For a review see D. Vollhardt, in Correlated Electron Systems, Proceedings of the Jerusalem Winter School of Theoretical Physics, edited by V. J. Emery (World Scientific, Singapore, 1993).

${ }^{7}$ M. Jarrell, H. Akhlaghpour, and Th. Pruschke, Phys. Rev. Lett. 70, 1670 (1993).

${ }^{8}$ M. Jarrell, Phys. Rev. B 51, 7429 (1995).

${ }^{9}$ A. Georges, G. Kotliar, W. Krauth, and M. J. Rozenberg, Rev. Mod. Phys. 68, 13 (1996).

${ }^{10}$ L. Craco and M. A. Gusmão, Phys. Rev. B 52, 17135 (1995).

${ }^{11}$ L. Craco and M. A. Gusmão, Phys. Rev. B 541629 (1996).
${ }^{12}$ L. M. Falicov and J. C. Kimball, Phys. Rev. Lett. 22, 997 (1969).

${ }^{13}$ U. Brandt and C. Mielsch, Z. Phys. B 75, 365 (1989).

${ }^{14}$ P. G. J. van Dongen and D. Vollhardt, Phys. Rev. Lett. 65, 1663 (1990); V. Janis, Z. Phys. B 83, 227 (1991); V. Janis and D. Vollhardt, ibid. 91, 317 (1993).

${ }^{15}$ T. Takabatake et al., Phys. Rev. B 41, 9607 (1990); M. F. Hundley et al., ibid. 42, 6842 (1990); A. Severing et al., ibid. 44, 6832 (1991).

${ }^{16}$ Other cumulant expansions of the PAM had appeared in the literature. See, for example, M. S. Figueira, Me. E. Foglio, and G. G. Martinez, Phys. Rev. B 50, 17993 (1994).

${ }^{17}$ Th. Pruschke, D. L. Cox, and M. Jarrell, Phys. Rev. B 47, 3553 (1993).

${ }^{18}$ M. B. Maple, M. C. de Andrade, J. Herrmann, Y. Dalichaouch, D. A. Gajewski, C. L. Seaman, R. Chau, R. Movshovich, M. C. Aronson, and R. Osborn, J. Low Temp. Phys. 99, 223 (1995).

${ }^{19}$ L. Degiorgi, H. R. Ott, and F. Hulliger, Phys. Rev. B 52, 42 (1995). 